\title{
Education-Oriented Virtual Environment for Clothing Thermal Functional Performance
}

\author{
Xiaonan $\mathrm{Luo}^{1}$, Wenbang $\mathrm{Hou}^{1}$, and $\mathrm{Yi}^{2}{ }^{2}$ \\ ${ }^{1}$ Computer Application Institute, SUN YAT-SEN University, \\ Guang Zhou 510275, China \\ ${ }^{2}$ Institute of Textiles and Clothing, The Hong Kong Polytechnic University, \\ Hung Hom, Hong Kong \\ Inslxn@zsu.edu.cn, houwenbang@yahoo.com.cn, \\ tcliyi@inet.polyu.edu.hk
}

\begin{abstract}
This paper presents a simulation environment for the education of clothing thermal function. It allows learners in a virtual environment to learn the clothing thermal comfort theory and identify the most important factors that influence clothing thermal functional performance. The virtual wearing case is defined with primary parameters and the numerical simulation results are visualized with 3D virtual environment and 2D animation, which provide virtual reality based interpretation of the thermal state and the dynamic thermal processes of the human-clothing system. This system can also be used as an effective visualization tool by textile researchers.
\end{abstract}

Keywords: Visualization; education oriented; clothing thermal functional performance; virtual environment.

\section{Introduction}

The thermal functional performance is a critical aspect that is highly concerned in clothing design. Especially during bad thermal conditions, the thermal protection of clothing relates to the health and survival of human beings. Even in ordinary living conditions, there are more and more consumers begin to concern the thermal comfort performance of clothing.

To explore the factors and mechanisms that affect the thermal functional performance of clothing, many advances have been achieved. In recent years, researchers have made great efforts to develop numerical models such as the thermoregulation of human body, the heat and moisture transfer behaviors of clothing material, as well as the dynamical interaction between human, clothing and environment system [1], [2], [3]. Based on the numerical models, Li et al proposed a virtual system for clothing thermal functional design [4], which can design clothing and analyze its thermal functional performance effectively. Although this system provides GUI interface and good 3D visualization for the result dataset, it's still very difficult and inconvenient for education of students and young engineers in clothing industry because of so many kinds of professional terms and intrinsic 
complexity of numerical solver. It's would be valuable to develop a learning tool that can educate people for a dedicated clothing, what will the thermal effect like in different wearing cases, how does the thermal processes interact in human-clothing system, how does the temperature and dampness distribute in every clothing layers, and what are the key factors that decide the thermal function of clothing.

In most cases, simulation environments are powerful learning tools and have been used in many engineering fields successfully [5-7]. Such systems are generally based on sophisticated didactical concepts and have used the modern technical possibilities include 3D visualization and virtual reality technique. They can enable exploration by allowing learners to manipulate parameters and visualize results in a virtual reality environment. In general there are two basic kinds of such systems: inanimate (offline) and live (on-line, real-time) [8]. Inanimate simulators are used to evaluate complex equations and models. They do not simulate real-time operations of physical systems; therefore the user interaction is limited. While live simulators are highly interactive and allowing learners to explore, they are always very complicated and time-consuming. To overcome their shortcuts, it's necessary to find an appropriate approach that allows the utmost manipulation options and decreases the complexity and time consumption simultaneously.

In this paper, we present a simulation based learning tool for clothing thermal functional performance. It could be called "half-live" simulation because the simulation is completed in advance and the result dataset is stored in database. By reorganizing the input data of simulation cases, user can manipulate parameters and explore situations in a more instinct and simple way as if by imitating a simulation case. This program can visualize simulation datasets in a virtual environment, and special attention is given to the design of a user interface being easy to learn and to use. The rest of the paper is organized as follows: Section 2 describes the numerical model for clothing thermal function simulation, and section 3 presents the details of the "half-live" simulation design. The visualization techniques are described in Section 4, and examples are presented in Section 5. Finally, we conclude and point out the future works.

\section{Numerical Model for Clothing Thermal Function Simulation}

The key factors that influence the thermal functional performance include the thermal properties of clothing materials (Fiber, membrane, PCM et al.), and the structure features of clothing assemblies. In the virtual design system, all materials can be designed and stored in database, even include the human body and environment conditions [4]. The specified human body, clothing and environment together consist a simulation scenario, and one or more scenarios with respective phase time comprise a simulation case. After all definition completed, the thermal processes in human bodyclothing-environment system, as shown in Fig. 1, are simulated, and the dynamic thermal state of human body and clothing are calculated as the result datasets. 


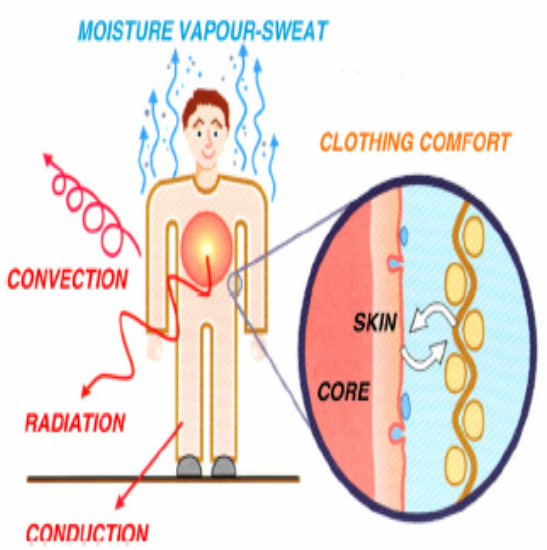

Fig. 1. Thermal processes in Human-Clothing- Fig. 2. Primary parameters used to define simulaEnvironment system

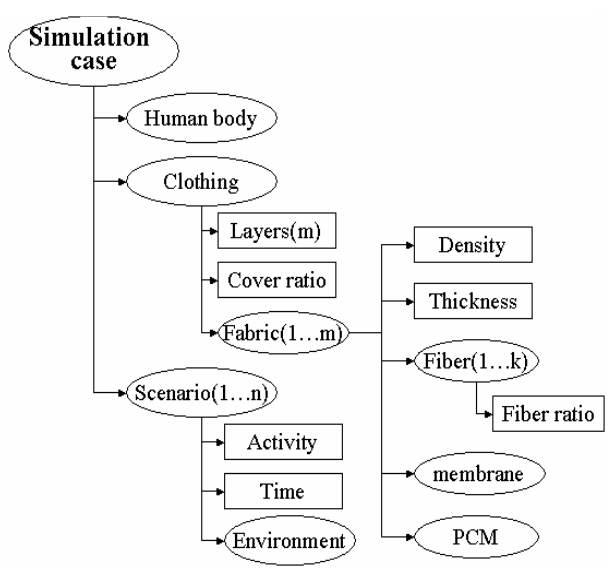

tion case

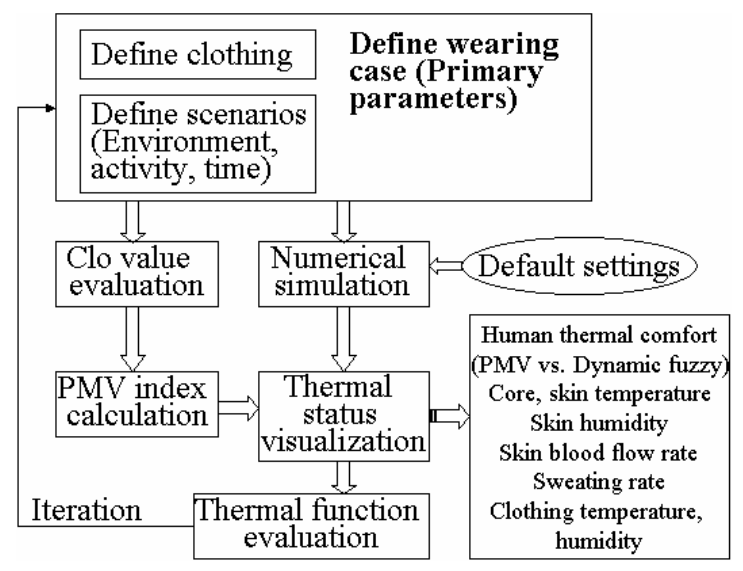

Fig. 3. System architecture of the half-live simulation

\section{The Half-Live Simulation}

As hereinbefore analysis, the numerical models have many kinds of parameters and most of them are professional nomenclatures that are difficult and impossible to give an instinct perception. To make the virtual environment as realistic as possible, only the primary and perceptual parameters are used to construct the simulation cases. These primary parameters are input by graphical icons to enhance the virtual environment. To retrieve the relative simulation dataset with the input primary parameters, the definition data of simulation cases are well organized. The primary parameters and the simulation case definition are shown as in Fig. 2. 
Because this half-live simulation is based on the existed simulation result, it's significant to assure that all reasonable simulation cases can be retrieved. Considering the fact that the available clothing materials, the effect clothing assemblies, the human body properties and the typical environment conditions are all limited and predictable, most of the meaningful simulation cases can be designed and calculated preliminarily. In case that no exact matched cases can be found, it always can find the nearest case and would not lead any big impact for learning purpose. The system is also designed to be able to collect such case definitions for appending its simulation result afterwards. The system architecture is shown as in Fig. 3.

\section{Visualization Overview}

\subsection{Virtual Environment}

In order to visualize the thermal state of a clothed human being as realistic as possible, 3D real geometrical models are used to construct a virtual scene, and the 2D animation as a supplement. Human bodies and clothing sets models exported from Poser@ 5 are used to visual the clothed people. They can express some important parameters such as body mass, surface area, clothing cover ratio and clothing style approximately. The male character model is realistic enough with about 35,000 triangular faces. For environment visualization, a virtual climate chamber is designed with wall color representing the air temperature, lighting on the wall representing the radiant temperature, a hygrometer on the wall to reflect the relative humidity, and the waving window curtain for air velocity.

The thermal state of a clothed human being include many kinds of parameters such as human thermal comfort, body temperature, skin humidity, sweating rate, metabolic rate and blood flow rate, as well as the temperature and humidity of each clothing layer. It's necessary to display these parameters simultaneously to explore the thermal processes and thermal state more accurately. To achieve this aim, the $2 \mathrm{D}$ animation and cross section view are provided additionally.

\subsection{Perceptual Colormap}

Visualization is a process of mapping data onto graphical primitives like colored sets of lines or polygons. A proper selection of colormap is important to produce an effective visualization. The most common rainbow colormap is typically the default map. Although it has the problem that the minimum perceivable increment varies widely over the range of the map $[10,11]$, it is still ubiquitous in scientific visualization for following reasons: It is attractive, colorful, with easy to discriminate "regions" of the data which can then be associated with specific data values given a colorbar key (low values with blue, medium-low with cyan, medium with green, etc.) [12]. Therefore the rainbow colormap is used to map the temperature data with improvement from 2 respects. One is the correction of using several piecewise linear maps instead of a single linear map between blue-green-red color space. The other is by fixing the scale points of temperature that reflect the human thermal sensations such as hot, warm, cool and cold. In general, red is for hot, yellow to green for warm, 


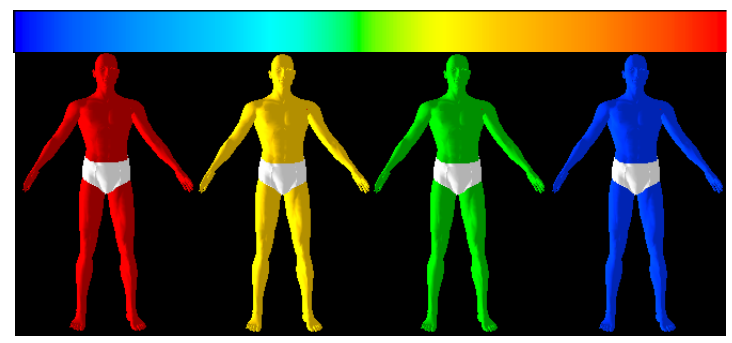

Fig. 4. Colormap examples, top - the improved rainbow colormap for temperature; bottomfrom left to right, color maps for hot, warm, cool and cold

green to light blue for cool, and blue for cold. For humidity, a luminance blue colormap is used. The designed colormap examples are shown as in Fig. 4.

\subsection{Adaptive Exploring for Time-Varied Data}

In order to generate the virtual environment with maximum realistic, it's necessary to realize the real-time animation of the dynamic thermal processes in human-clothing system, thereby it can help learners to sense the change speed of body thermal state in a defined wearing case. On the other hand, the actual time period of the simulation case maybe too long to wait, it's will helpful to provide a flexible control on animation speed.

Real-time animation. To realize real-time clock step, the clock event of windows Timer control is used. The calculation formula for real time is

$$
t=t_{0}+\left(T-T_{0}\right) * n
$$

where:

$\mathrm{t}$ - real simulation time.

$\mathrm{t}_{0}$ - previous end simulation time (considering the pause operation).

$\mathrm{T}$ - current computer system time.

$\mathrm{T}_{0}$ - computer system time when start.

$\mathrm{n}-$ animation speed, $\mathrm{n}=1$ means real time.

Adaptive time-varied data exploring. In most situations, the analysis may care more on quick varying data zone instead of whole simulation period. To automatically display those remarkable data zone, an adaptive algorithm is used. For a given parameters, first a valve value is set, then for each time step the datum is compared with this value, and is displayed only if it's larger than the valve value.

\section{Example}

In order to illustrate the system functions and the evaluation process of clothing thermal functional performance, a wearing case with contrastive definitions are introduced. The dynamical thermal states are visualized and the traditional thermal 
comfort index - PMV index is calculated for reference. As shown in Fig. 5, the wearing case definition interface consists of a tree structure navigator and a tree-node context-sensitive detail form. Each wearing case is the root node, and some of the sub-nodes can be added or deleted dynamically. When a sub-node is selected, the relative detail definition form will be activated.

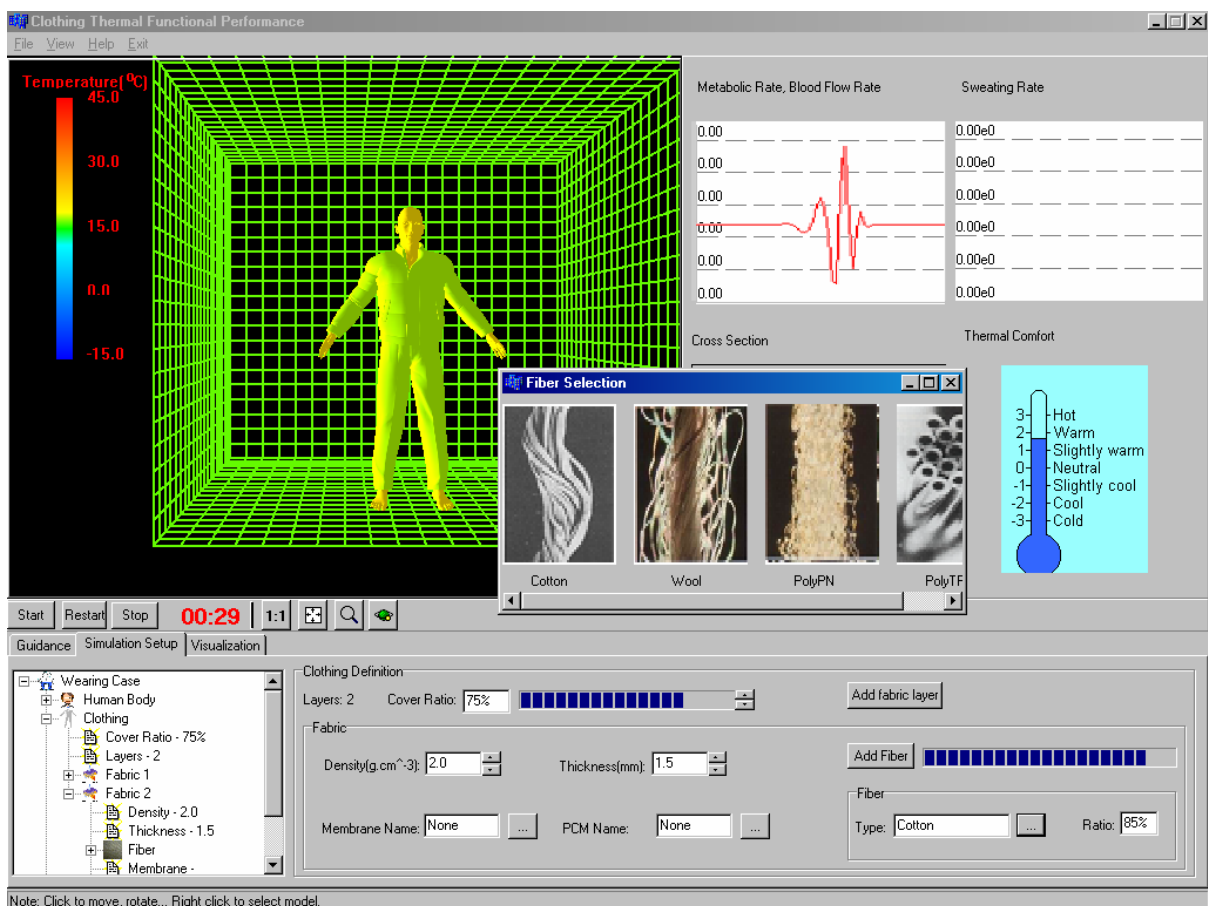

Fig. 5. Wearing case definition: left is the wearing case definition tree, right is a detailed definition page that related to the selected tree node

The wearing case is for summer outdoor walking with three clothing layers, the definition is as follows:

Human body specification: Resting (1 Met), walking (2 Met).

Environmental specification: $35{ }^{\circ} \mathrm{C}$ and $80 \%$ relative humidity, air conditional room is $25^{\circ} \mathrm{C}$ and $60 \%$ relative humidity.

Clothing design: three cotton fabrics layer.

Scenario: rest for $15 \mathrm{~min}$. in room, then walking for $15 \mathrm{~min}$.

Figure 6 shows snap shot from the thermal processing animation for summer wearing case. Temperatures of body skin and the outside fabric layer are visualized in virtual scene, the cross section view for displaying detail temperature distribution, and the 2D animation for body thermal physiological parameters. It's evident that the clothing with three layers has hot thermal functional performance when walking in summer thermal condition. 


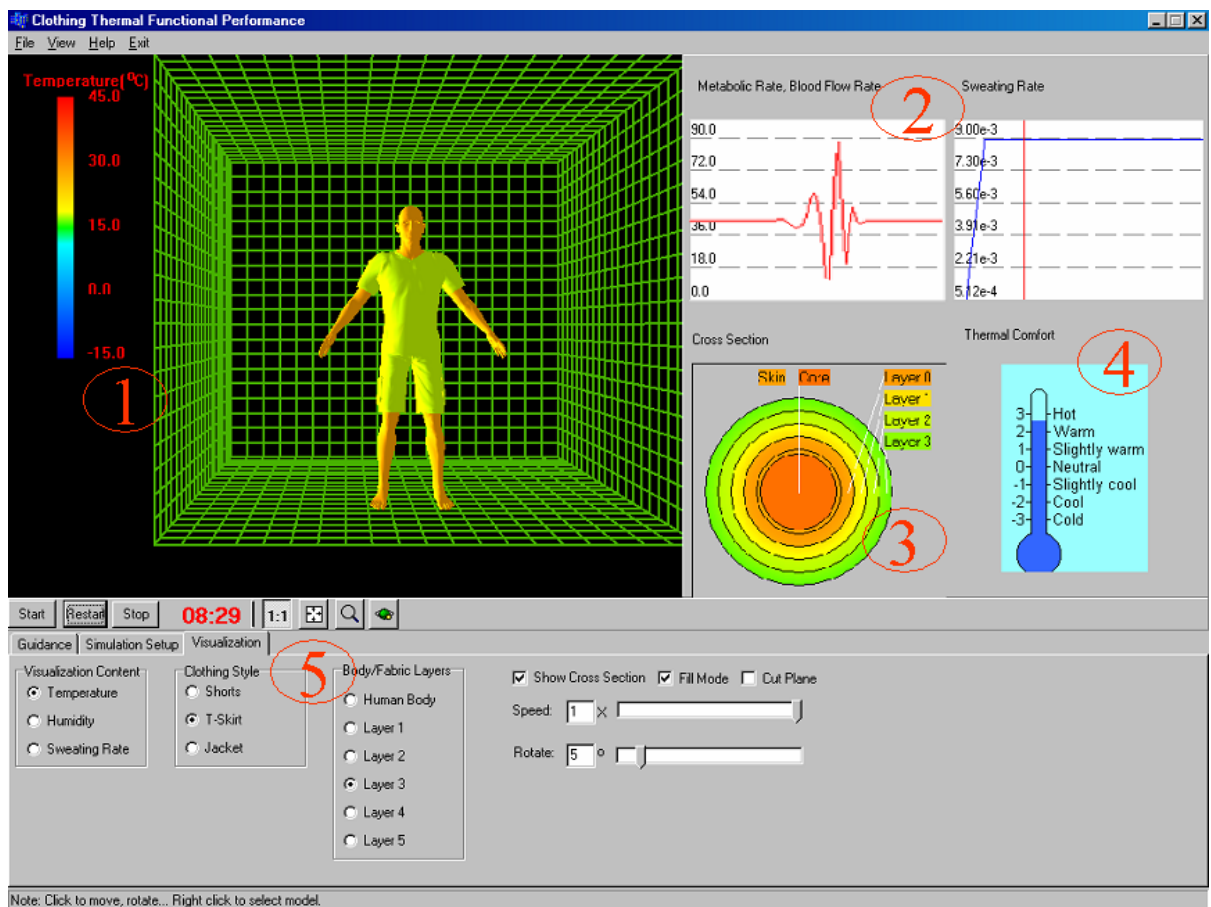

Fig. 6. Thermal state visualization: region $1-3 D$ virtual scene; region $2-2 D$ animation; region 3 - cross section; region 4 - thermal comfort index; and region 5 - visualization control

\section{Conclusions and Future Work}

This paper reports the development of a virtual environment for the education of clothing thermal function. The virtual wearing case is defined with primary parameters and the numerical simulation results are visualized with $3 \mathrm{D}$ virtual environment and 2D animation, which interpret the thermal state and the dynamic thermal processes of the human-clothing system in a vivid mode. It allows learners in a virtual environment to learn the clothing thermal comfort theory and identify the most important factors that influence clothing thermal functional performance. This virtual environment system is specially developed to help teaching clothing thermal functional design for students and new designers, and it can also be used as a visualization tool by textile researchers. The trial use proves that this system can provide good demonstration of clothing thermal function model numerical simulation result, giving user an intuitive and insightful understanding of clothing thermal function and thermal comfort.

Further works are to implement the virtual reality scene and behavior animation, and to develop an Internet application version using VRML models. 
Acknowledgments. This research is supported by the National Science Fund for Distinguished Young Scholars (No.60525213) and the Key Project (No. 60533030) of NSFC, and 973 Program of China (No.206CB303106).

\section{References}

1. Wang, Z., Li, Y.: An Integrative Adaptive Model for Simulating Human Dynamic Thermal Comfort in the Body-Clothing-Environment System. Psychophysiology (2003)

2. Li, Y., Li., F., Liu, Y.X., Luo, Z.X.: An integrated model for simulating interactive thermal processes in human-clothing system. Journal of Thermal Biology 29 (2004) 567-575

3. Gagge, A.P., Stolwilk, J.A.J., Nishh, Y.: An Effective Temperature Scale Based on a Simple Model of Human Physiological Regulation Response. ASHRAE Trans. 77 (1971) 247-262

4. Li, Y. et al.: P-smart-a virtual system for clothing thermal functional design. ComputerAided Design 38 (2006) 726-739

5. Jentsch, M., Reimann, K.: E-Learning for Structural Analysis. Proceedings of the 20th CAD-FEM Users' Meeting 2002 International Congress on FEM Technology, Germany (2002)

6. Ostermann, T. et al.: eLearning Platform with Microelectronic Contents. Proceedings of the IASTED International Conference on WEB-BASED EDUCATION - WBE 2004, Innsbruck, Austria (2004)

7. Mokhtar, A., Khan, M.: Education - oriented visualization model for buildings cross ventilation. Proceedings of the 2nd international conference on Computer graphics and interactive techniques in Australasia and South East Asia, Singapore (2004) 71-73

8. Nahvi, M.: Dynamics of student-computer interaction in a simulation environment: Reflections on curricular issues. Proceedings of Frontiers in Education'96, IEEE, 13831386

9. ISO: ISO 7730 Moderate Thermal Environments - Determination of the PMV and PPD indices and specification of the conditions for thermal comfort. 2nd ed. International Organization for Standardization, Geneva, Switzerland (1995)

10. Rogowitz, B.E., Treinish, L.: Data Visualization: The End of the Rainbow. IEEE Spectrum 35 (1998) 52-59

11. Bergman, L.D., Rogowitz, B.E., Treinish, L.A.: A rule-based tool for assisting colormap selection. Proceedings of IEEE Visualization (1995) 118-125

12. Kalvin, A. D., Rogowitz, B. E., Pelah, A., Cohen, A.: Building perceptual color maps for visualizing interval data. Proceedings of SPIE Conference on Human Vision and Electronic Imaging, San Jose, CA. (2000) 323-335 